\title{
1, Semiglobal Cototal Domination on Graphs
}

\author{
T. Nicholas ${ }^{1}$, T. Sheeba Helen ${ }^{2}$ \\ ${ }^{1}$ Department of Mathematics, St.Judes College,Thoothoor, TamilNadu, India \\ ${ }^{2}$ Department of Mathematics, Holy Cross College (Autonomous), Nagercoil - 4, TamilNadu, India
}

\begin{abstract}
A subset $D$ of vertices of a connected graph $G$ is called a semiglobal cototal dominating set if $D$ is a dominating set for $G$ and $G^{\text {sc }}$ and $\left\langle V-D>\right.$ has no isolated vertices in $G$, where $G^{\text {sc }}$ is the semi complementary graph of $G$. The semiglobal cototal domination number is the minimum cardinality of a semiglobal cototal dominating set of $G$ and is denoted by $\gamma_{\text {sgcot }}(G)$. In this paper we initiate a study of this new parameter $\gamma_{\text {sgcot }}(G)$. Some bounds on this parameter are obtained and their exact values for some standard graphs are established.
\end{abstract}

\section{Subject Classification: 05C69}

Keywords: Semicomplete graph, global cototal domination number, semicomplementary graph, semiglobal cototal domination number

\section{Introduction}

All graphs considered in this paper are simple, finite, undirected and connected. For all graph theoretic terminology not defined here, the reader is referred to [1]. For a comprehensive introduction to theoretical and applied facts of domination in graphs the reader is directed to the book [2].

A set $D$ of vertices is called a dominating set of $G$ if each vertex not in $D$ is joined to some vertex in $D$. The domination number $\gamma(\mathrm{G})$ is the minimum cardinality of the dominating set of $\mathrm{G}[2]$.

A dominating set $\mathrm{D}$ is called a global dominating set of $\mathrm{G}$ if $D$ is a dominating set of both $G$ and $G^{c}$. The global domination number of $\mathrm{G}$, denoted by $\gamma_{\mathrm{g}}(\mathrm{G})$ is the minimum cardinality of the global dominating set of $G$ [6]. A dominating set $D$ of a connected graph $G$ is called a connected dominating set of $\mathrm{G}$ if the induced subgraph $\langle\mathrm{D}\rangle$ is connected. The connected domination number of $G$, denoted by $\gamma_{c}(G)$ is the minimum cardinality of the connected dominating set of G [7]. A dominating set D of a connected graph is called a independent dominating set of $G$ if the induced subgraph $\langle\mathrm{D}\rangle$ is a null graph [2].

$\mathrm{G}$ be a connected graph, then the semi complementary graph of $G$, denoted by $G^{\text {sc }}$, has the same vertex set as that of $G$ and has edge set $\{\mathrm{uv} / \mathrm{u}, \mathrm{v} \in \mathrm{V}(\mathrm{G})$, uv $\rightleftarrows E(G)$ and there is $\mathrm{w} \in \mathrm{V}(\mathrm{G})$ such that uw,wv $\in \mathrm{E}(\mathrm{G})\}$ [4]. Let $\mathrm{G}$ be a connected graph. Then $G$ is said to be semi complete if any pair of vertices in $G$ have a common neighbor. The necessary and sufficient condition for a connected graph to be semicomplete is that any pair of vertices lie on the same triangle or lie on two triangles having a common vertex [4].

Recently Nicholas and Sheeba Helen introduced the concept global cototal domination number in [5]. A dominating set D of a graph $\mathrm{G}$ is a global cototal dominating set if $\mathrm{D}$ is both a global dominating set and a cototal dominating set. The global cototal domination number $\gamma_{\mathrm{gcot}}(\mathrm{G})$ is the minimum cardinality of a global cototal domination set of $\mathrm{G}$.
A new graph parameter, the semiglobal domination number, for a connected graph $G$ was introduced by Siva Rama Raju and Kumar Addagarla in [9]. A dominating set $\mathrm{D} \subseteq \mathrm{V}(\mathrm{G})$ is called a semiglobal dominating set(sgd-set) of $G$, if $D$ is a dominating set for $G$ and $G^{\text {sc }}$. The semiglobal domination number is the minimum cardinality of a semiglobal dominating set of $\mathrm{G}$ and is denoted by $\gamma_{\mathrm{sg}}(\mathrm{G})$.

The purpose of this paper is to study the semiglobal cototal domination number, for a connected graph G. We call D $\subseteq$ $\mathrm{V}(\mathrm{G})$ a semiglobal cototal dominating set, if $\mathrm{D}$ is a dominating set for $G$ and $G^{\text {sc }}$ and $\langle V-D\rangle$ has no isolated vertices in $G$, where $G^{\text {sc }}$ is the semicomplementary graph of G. The semiglobal cototal domination number is the minimum cardinality of a semiglobal cototal dominating set of $\mathrm{G}$ and is denoted by $\gamma_{\mathrm{sgcot}}(\mathrm{G})$.

In this paper some bounds on this parameter are obtained and their exact values for some standard graphs like cycle, path, complete graph, complete bipartite graph, star and crown graph are established.

\section{Main Results}

Proposition 1.1 For the cycle $C_{n}, n \geq 6$

$\gamma_{\mathrm{sgcot}}\left(\mathrm{C}_{\mathrm{n}}\right)= \begin{cases}\frac{n}{3}, & n \equiv 0(\bmod 3) ; \\ \left\lceil\frac{n}{3}\right], & n \equiv 1(\bmod 3) ; \\ \left\lceil\frac{n}{3}\right\rceil+1, & n \equiv 2(\bmod 3) .\end{cases}$

Proof: Let $\mathrm{V}\left(\mathrm{C}_{\mathrm{n}}\right)=\left\{\mathrm{v}_{0}, \mathrm{v}_{1}, \mathrm{v}_{2}, \mathrm{v}_{3}, \ldots, \mathrm{v}_{\mathrm{n}-1}\right\}$ and

$\mathrm{E}\left(\mathrm{C}_{\mathrm{n}}\right)=\left\{\mathrm{v}_{\mathrm{i}} \mathrm{v}_{\mathrm{i}+1} / \mathrm{i}=0,1,2, \ldots, \mathrm{n}-1\right\}$, subscript modulo $\mathrm{n}$.

If $\mathrm{G}=\mathrm{C}_{\mathrm{n}},(\mathrm{n} \geq 6)$ then $C_{n}^{\Delta c}=\left\{\begin{array}{l}\frac{C_{\frac{n}{2}} \cup C_{\frac{n}{2}} \text { if } n \text { is even; }}{C_{n}} \text { if } n \text { is odd. }\end{array}\right.$

Let $\mathrm{D}$ be a minimal semi global cototal dominating set of $\mathrm{C}_{\mathrm{n}}$. Let $v_{i} \in D$. Then $i+3 \leq n$ is the least positive integer such that $v_{i}, v_{i+3} \in D$. Hence the result follows. 


\section{International Journal of Science and Research (IJSR) \\ ISSN (Online): 2319-7064}

Index Copernicus Value (2015): 78.96 | Impact Factor (2015): 6.391

Proposition $1.2 \gamma_{\mathrm{sgcot}}\left(\mathrm{K}_{\mathrm{n}}\right)=\mathrm{n}, \mathrm{n} \geq 3$.

Proof: All the vertices are isolated in the semicomplementary graph of the complete graph. Therefore, the semiglobal cototal dominating set must contain all the vertices of $K_{n}$.

Hence $\gamma_{\text {sgcot }}\left(K_{n}\right)=n, n \geq 3$.

Proposition $1.3 \gamma_{\mathrm{sgcot}}\left(\mathrm{K}_{\mathrm{m}, \mathrm{n}}\right)=2 \mathrm{~m}, \mathrm{n} \geq 2$

Proof: Let $V_{1}$ and $V_{2}$ be the partite sets of $K_{m, n}$ with $\left|V_{1}\right|=\mathrm{m},\left|V_{2}\right|=\mathrm{n}$. Every vertex in a partite set dominates every other vertex of the other. Then $D=\left\{u_{i}, v_{j}\right\}$ is a minimal dominating set for $\mathrm{K}_{\mathrm{m}, \mathrm{n}}$ where $\mathrm{u}_{\mathrm{i}} \in \mathrm{V}_{1}, \mathrm{v}_{\mathrm{j}} \in \mathrm{V}_{2}$, for some $\mathrm{i}$ and $\mathrm{j}$. Then the induced subgraph $\langle\mathrm{V}-\mathrm{D}\rangle$ has no isolated vertex. The semicomplementary graph of the complete bipartite graph $\mathrm{K}_{\mathrm{m}, \mathrm{n}}$ is a disconnected graph $\mathrm{K}_{\mathrm{m}} \mathrm{UK}_{\mathrm{n}}$, where $\left\langle\mathrm{V}_{1}\right\rangle=\mathrm{K}_{\mathrm{m}}$ and $\left\langle\mathrm{V}_{2}\right\rangle=\mathrm{K}_{\mathrm{n}}$. Any two vertices in $\mathrm{V}_{1}$ or that of $\mathrm{V}_{2}$ are adjacent in $K_{m, n^{*}}^{s c}$ Hence $\mathrm{D}=\left\{\mathrm{u}_{\mathrm{i}}, \mathrm{V}_{\mathrm{j}}\right.$ \} is a dominating set for the semicomplementary graph of $\mathrm{K}_{\mathrm{m}, \mathrm{n}}$. Thus $\gamma_{\mathrm{sgcot}}\left(\mathrm{K}_{\mathrm{m}, \mathrm{n}}\right)=2, \mathrm{~m}, \mathrm{n} \geq 2$.

Proposition $1.4 \gamma_{\mathrm{sgcot}}\left(K_{1, n}\right)=n+1, n \geq 3$.

Proof: Let $\mathrm{V}(\mathrm{G})=\left\{\mathrm{v}, \mathrm{v}_{1}, \mathrm{v}_{2}, \mathrm{v}_{3}, \ldots, \mathrm{v}_{\mathrm{n}}\right\}$, where $\mathrm{v}$ is the only vertex of degree $n$ and each $v_{i}$ is a pendant vertex adjacent to v. The semicomplementary graph of $K_{1, n}$ is a disconnected graph $K_{1} \cup K_{n}$. Hence $D=V(G)$ is the semiglobal cototal dominating set of $K_{1, n}$.

Thus $\gamma_{\mathrm{sgcot}}\left(K_{1, n}\right)=n+1, n \geq 3$.

The crown graph $\mathrm{C}_{\mathrm{n}} \odot \mathrm{K}_{1}$ is the graph obtained from cycle $\mathrm{C}_{\mathrm{n}}$ by attaching a pendant edge to each vertex of the cycle.

Proposition $1.5 \gamma_{\mathrm{sgcot}}\left(\mathrm{C}_{\mathrm{n}} \odot \mathrm{K}_{1}\right)=\mathrm{n}$, where $\mathrm{n}$ is the length of the cycle.

Proof: Let $G=C_{n} \odot K_{1} \cdot V(G)=\left\{v_{0}, v_{1}, v_{2}, \ldots, v_{n-1}\right\} \cup\{$ $\left.\mathrm{u}_{0}, \mathrm{u}_{1}, \mathrm{u}_{2}, \ldots, \mathrm{u}_{\mathrm{n}-1}\right\}$.

$\mathrm{E}(\mathrm{G})=\left\{\mathrm{v}_{\mathrm{i}} \mathrm{v}_{\mathrm{i}+1} / \mathrm{i}=0,1,2, \ldots, \mathrm{n}-1\right.$, subscript modulo $\left.\mathrm{n}\right\}$

$U\left\{\mathrm{u}_{\mathrm{i}} \mathrm{v}_{\mathrm{i}} / \mathrm{i}=0,1,2, \ldots, \mathrm{n}-1\right\}$. Since the minimal semiglobal cototal dominating set $\mathrm{D}$ consists of all the $n$ vertices of $K_{1}$ in $C_{n} \odot K_{1}$, $\left\langle V-D>=C_{n}\right.$, has no isolated vertex. Thus $\gamma_{\text {sgcot }}\left(C_{n} \odot K_{1}\right)=n$.

Proposition 1.6 For a path $\mathrm{P}_{\mathrm{n}}$ on $\mathrm{n}$ vertices,

$$
\mathrm{Y}_{\mathrm{sgcot}}\left(\mathrm{P}_{\mathrm{n}}\right)=\left\{\begin{aligned}
\frac{n}{3}+2, & n \equiv 0(\bmod 3) ; \\
\frac{n+2}{3}, & \equiv 1(\bmod 3) ; \\
\left\lceil\frac{n}{3}\right\rceil+1, n & \equiv 2(\bmod 3) .
\end{aligned}\right.
$$

Proof: Let $P_{n}$ be the path of order $n . V\left(P_{n}\right)=\left\{v_{0}, v_{1}, v_{2}, v_{3}\right.$, . , $\left.\mathrm{v}_{\mathrm{n}-1}\right\}$.

If $\mathrm{G}=\mathrm{P}_{\mathrm{n}}(\mathrm{n} \geq 3)$ then $\mathrm{G}^{\mathrm{sc}}=P_{\frac{\mathrm{n}}{\mathrm{z}}} \cup P_{\frac{\mathrm{n}}{\mathrm{z}}}$ if $\mathrm{n}$ is even

$=\frac{P_{n+1}}{\mathrm{n}} \cup \frac{P_{\mathrm{n}-1}}{\mathrm{n}}$ if $\mathrm{n}$ is odd

Since $D$ is a semiglobal cototal dominating set in $G, i+3$ $\leq n$ is the least positive integer such that $v_{i}, v_{i+3} \in D$. D must contain $\mathrm{v}_{0}$ and $\mathrm{v}_{\mathrm{n}-1}$, the end vertex of $\mathrm{P}_{\mathrm{n}}$.

If $\mathrm{n} \equiv 0(\bmod 3)$ then $\mathrm{D}$ contains $\mathrm{v}_{3 \mathrm{i}}$ where $\mathrm{i}=0,1, \ldots$, $\frac{n-3}{3}$ and the vertices $\mathrm{v}_{\mathrm{n}-2}$ and $\mathrm{v}_{\mathrm{n}-1}$. Hence $|D|=\frac{n-3}{3}+1+2=\frac{n}{3}+2$.

If $\mathrm{n} \equiv 1(\bmod 3)$ then $\mathrm{D}$ contains $\mathrm{v}_{3 \mathrm{i}}$ where $\mathrm{i}=0,1, \ldots$, $\frac{n-1}{3}$. Thus $|D|=\frac{n-1}{3}+1=\frac{n+2}{3}$.

If $\mathrm{n} \equiv 2(\bmod 3)$ then $\mathrm{D}$ has $\mathrm{v}_{3 \mathrm{i}}$ where $\mathrm{i}=0,1, \ldots, \frac{n-2}{3}$ and also $\mathrm{v}_{\mathrm{n}-1}$.

In this case $|D|=\frac{n-2}{3}+1+1=\frac{n+1}{3}+1=\left\lceil\frac{n}{3}\right\rceil+1$

Hence the result follows.

Theorem1.7 If $G$ is a connected graph and $\gamma_{\text {sgcot }}(G)=2$, then (i)There is an edge uv in $\mathrm{G}$ such that each vertex inV $-\{\mathrm{u}, \mathrm{v}\}$ is adjacent to $\mathrm{u}$ or $\mathrm{v}$ but not both.

(ii) There is a path $\mathrm{P}_{4}$, each vertex in $\mathrm{V}-\mathrm{V}\left(\mathrm{P}_{4}\right)$ lies on an edge whose end vertices are dominated by end vertices of $\mathrm{P}_{4}$.

Proof: Suppose that $\gamma_{\text {sgcot }}(G)=2$. Without loss of generality assume that $\mathrm{D}=\{\mathrm{u}, \mathrm{v}\}$ is a minimal semiglobal cototal dominating set in $\mathrm{G}$.

Case (i) $<\mathrm{D}>$ is connected in $\mathrm{G}$

Clearly uv is an edge in G. If any vertex $\mathrm{w}$ in $\mathrm{V}-\{\mathrm{u}, \mathrm{v}\}$ is adjacent to both $\mathrm{u}$ and $\mathrm{v}$, then $\mathrm{D}$ is not a dominating set for $\mathrm{G}^{\mathrm{sc}}$. Hence (i) holds.

Case (ii) $<\mathrm{D}>$ is not connected in $\mathrm{G}$.

No vertex in V-D is adjacent to both $u$ and $v$. Hence there is a path $\mathrm{P}_{4}$ from $\mathrm{u}$ to $\mathrm{v}$ in $\mathrm{G}$, say $\mathrm{uv}_{1} \mathrm{v}_{2} \mathrm{v}$ Let $\mathrm{v}_{3} \in \mathrm{V}-\mathrm{V}\left(\mathrm{P}_{4}\right)$. Since $D$ is a $\gamma_{\text {sgcot }}$ set in $G, v_{3}$ is adjacent to $u$ or $v$ in $G$ but not both. For $v_{3}$ to be dominated by a vertex in $\mathrm{D}, \mathrm{v}_{3}, \mathrm{v}$ are to be connected by a path of length two in $G$, say $v_{3} v_{4} v$. Hence $v_{3}$ lies on an edge $v_{3} v_{4}$ and $v_{3}, v_{4}$ are dominated by $u$ and $\mathrm{v}$ (end vertices in $\mathrm{P}_{4}$ ) respectively. Hence (ii) holds

Note: Every semiglobal cototal dominating set for $G$ is a global cototal dominating set for $\mathrm{G}$, but the converse is not true. That is, $\gamma_{\text {gcot }}(G) \leq \gamma_{\text {sgcot }}(G)$.

Similarly, every semiglobal cototal dominating set for $G$ is also a semiglobal dominating set for $\mathrm{G}$, but the converse is not true. That is, $\gamma_{\text {sg }}(\mathrm{G}) \leq \gamma_{\text {sgcot }}(\mathrm{G})$. 


\section{International Journal of Science and Research (IJSR) \\ ISSN (Online): 2319-7064}

Index Copernicus Value (2015): 78.96 | Impact Factor (2015): 6.391

Theorem1.8 Let $E^{b}$ be the set of all independent edges of $\mathrm{K}_{\mathrm{n}}$. Then

(i) $\gamma_{\mathrm{sgcot}}\left(\mathrm{K}_{\mathrm{n}}-E^{t}\right)=\frac{n+1}{2}$ when $\mathrm{n}$ is odd.

(ii) $\gamma_{\mathrm{sgcot}}\left(\mathrm{K}_{\mathrm{n}}-E^{\prime}\right)=\frac{n}{2}$ when $\mathrm{n}$ is even.

Proof: Let $\mathrm{K}_{\mathrm{n}}$ be a complete graph with n vertices. Let $E^{y}$ be the set of all independent edges.

Case(i) $n$ is odd.

Without loss of generality, let $E^{f}$ contain the independent edges from the outer cycle of $\mathrm{K}_{\mathrm{n}}$. Obviously $\left|E^{\prime}\right|=$ $\frac{n-1}{2}$. Then the semicomplementary of this graph contains $\left|E^{\prime}\right|$ number of $\mathrm{K}_{2}$ 's and an isolated vertex. Any one end vertex of each $\mathrm{K}_{2}$ and the isolated vertex form a semiglobal cototal dominating set

Hence $\gamma_{\mathrm{sgcot}}\left(\mathrm{K}_{\mathrm{n}}-E^{J}\right)=\left|E^{J}\right|+1=\frac{n-1}{2}+1=\frac{n+1}{2}$ when $\mathrm{n}$ is odd.

Case(ii) $n$ is even.

Here $\left|E^{y}\right|=\frac{n}{2}$. Then the semicomplementary of this graph contains a perfect matching, namely $\left|E^{y}\right| \mathrm{K}_{2}$. Any one end vertex of each $\mathrm{K}_{2}$ form a semiglobal cototal dominating set. Hence $\gamma_{\text {sgcot }}\left(\mathrm{K}_{\mathrm{n}}-E^{J}\right)=\left|E^{J}\right|=\frac{n}{2}$ when $\mathrm{n}$ is even.

Theorem1.9 If $G=K_{n}-\{e\}$, then $\gamma_{\text {sgcot }}(G)=n$.

Proof: Let $\mathrm{G}=\mathrm{K}_{\mathrm{n}}-\{\mathrm{e}\}$. Then $\mathrm{G}^{\mathrm{sc}}$ is a disconnected graph containing (n-2) $\mathrm{K}_{1} \cup \mathrm{K}_{2}$. Then any global dominating set will contain atleast $n-1$ vertices ( $n-2$ isolated vertices and one end vertex of $\mathrm{K}_{2}$ ). If $\mathrm{D}$ is a semi global cototal dominating set, then $|D| \geq \mathrm{n}-1$. If $|D|=\mathrm{n}-1$, then $\mathrm{D}$ contradicts the cototal domination property that $\langle\mathrm{V}-\mathrm{D}\rangle$ has no isolated vertex. Hence $|D|>\mathrm{n}-1$. Hence the theorem.

Theorem1.10 For a semicomplete graph $\mathrm{G}, \gamma_{\mathrm{sgcot}}(\mathrm{G}) \geq 3$.

Proof: Suppose the claim does not hold. Let $\gamma_{\text {sgcot }}(G)=2$

Let $D=\left\{v_{1}, v_{2}\right\}$ be a sgcot-d set in $G$.

Case(i) $\langle\mathrm{D}\rangle$ is connected in $\mathrm{G}$.

Then $v_{1} v_{2}$ is an edge in $G$. By the nature of semicomplete graph there is a $v_{3}$ in $G$ such that $\left\langle v_{1} v_{2} v_{3}\right\rangle$ is a triangle in $G$. This implies $D$ is not a dominating set in $G^{\text {sc }}$ which is a contradiction to $\mathrm{D}$ is a sgcot $\mathrm{d}$-set in $\mathrm{G}$.

Case(ii) $\langle D\rangle$ is disconnected in $G$.Since $G$ is semicomplete there is $v_{3}$ in $G$ such that $\left\langle v_{1} v_{3} v_{2}\right\rangle$ is a path in $G$. Then in $\mathrm{G}^{\mathrm{sc}}, \mathrm{v}_{3}$ is not dominated by any vertex in $\mathrm{D}$, a contradiction to $\mathrm{D}$ is a sgcot $\mathrm{d}$-set in $\mathrm{G}$. Hence in either case, we get a contradiction to $\mathrm{D}$ being a sgcot $\mathrm{d}$ - set in G. Since a semicomplete graph has at least a triangle $\gamma_{\text {sgcot }}(G) \neq 1$. This implies $\gamma_{\text {sgcot }}(\mathrm{G}) \geq 3$.

Theorem1.11 Let $\mathrm{G}$ be a connected graph such that $\delta(G)$ $\geq 2$ and $\mathrm{D}$ is an independent $\operatorname{sgcot} \mathrm{d}-$ set for $\mathrm{G}$. If there exists an independent set $D_{1}$ in V-D then $D_{1}$ is a sgcot d-set for $\mathrm{G}$.

Proof: Assume that $D_{1}$ is independent. Let $v \in D$. This implies that there is $v_{1}$ in $D_{1}$ such that $v_{1}$ is in $G$ (since $\delta(G) \geq 2$ ). Since $\mathrm{v}_{1}$ is in $\mathrm{D}_{1}$ and $\mathrm{D}$ is independent sgcot set in $G$ there is a $v_{2}$ in $D, v_{3}$ in $V$ such that $v_{1} v_{2} v_{3}$ is a path in G. Clearly $v_{3} \in D_{1}$. Since $D_{1}$ is independent, $v_{1} v_{2} v_{3}$ is a path in G. and $\mathrm{vv}_{3}$ is not an edge in G. For $v \in D$, there exists $v_{1} \in D_{1}$ such that $v_{v}$ is in $G$ and $v_{3}$ is in $G^{\text {sc }}$. Since $v$ is arbitrary, $D_{1}$ is sgcot $d$ - set in $G$.

\section{References}

[1] Harary, F. Graph Theory, Addison - Wesley, Reading, MA, 1972.

[2] T. W. Haynes, S. T. Hedetneimi, P. J. Slater, Fundamentals of Domination in Graphs, MarcelDekker, New York, 1988.

[3] V.R.Kulli, B. Janakiram and R.R Iyer. The cototal domination number of a graph J.Discrete Mathematical Sciences and Cryptography 2 (1999) 179-184.

[4] I. H. Naga Raja Rao, S. V. Siva Rama Raju, Semi Complementary Graphs, Thai Journal of Mathematics. Volume 12 (2014) number 1: 175 - 183

[5] T.Nicholas, T.Sheeba Helen, Global Cototal Domination in Graphs, Preprint.

[6] D. F. Rall, "Dominating a graph and its complement "Congr. Numer. , 80(1991), 89-95.

[7] E. SampathKumar, H. B. Walikar, The Connected Domination Number of a Graph, J. Math. Phy. Sci, 13(1979), $607-613$

[8] E. Sampathkumar, The global domination number of a graph J.MathPhys.Sci 23 (1989) 377-385

[9] S. V. Siva Rama Raju, S. S. V. R. Kumar Addagarla, Semiglobal Domination, International Journal of Mathematical Archive - 3(7), 2012, 2589 - 2593 